\title{
Network Representation and Passivity of Delayed Teleoperation Systems
}

\author{
Jordi Artigas, Jee-Hwan Ryu, Carsten Preusche, Gerd Hirzinger
}

\begin{abstract}
The paper proposes a general network based analysis and design guidelines for teleoperation systems. The electrical domain is appealing because it enjoys proficient analysis and design tools and allows a one step higher abstraction element, the network. Thus, in order to analyze the system by means of network elements the mechanical system must be first modeled as an electric circuit. Only then power ports become apparent and networks can be defined. This kind of analysis has been previously performed in systems with well defined causalities, specially in the communication channel. Indeed, a communication channel exchanging flow-like and effort-like signals, as for instance velocity and computed force, has a well defined causality and can thus be directly mapped as a two-port electrical network. However, this is only one of the many possible system architectures. This paper investigates how other architectures, including those with ambiguous causalities, can be modeled by means of networks, even in the lack of flow or effort being transmitted, and how they can be made passive for any communication channel characteristic (delay, package-loss and jitter). The methods are exposed in the form of design guidelines sustained with an example and validated with experimental results.
\end{abstract}

\section{INTRODUCTION}

The goal of the methods presented in this article is to facilitate the design of any possible teleoperation architecture on a systematic way, without the burden of having to explicitly tackle typical channel related issues, as are communication time delay, jitter and package loss. The network analysis [1] is one of the most accounted modeling frameworks for teleoperation systems [2], [3], [4], [5]. A network masks an electrical circuit, i.e. a collection of electrical components which accomplish a specific task. Sometimes it is convenient to speak of an electrical circuit as a network, de-emphasizing the internals of the circuit while stressing the interconnectivity medium, i.e. the port. Indeed, the port is a two-terminal interface which allows connectivity between networks and thus a transfer of energy. A power port is entirely represented by the pair of dual variables, current and voltage, whose product is power. Thus, the description of a system in terms of networks allows an energy based analysis and therefore allows to extract conclusions about passivity, a powerful tool for system stability.

The designs presented in this paper take place in an ideal channel scenario, where master and slave robots behave as rigidly connected masses, the communication delay is null and their power exchange is lossless. Stability out of the

Jordi Artigas, Carsten Preusche and Gerd Hirzinger are with the Institute of Robotics and Mechatronics at the German Aerospace Center, 82234 Wessling, Germany.

J.H. Ryu is Faculty of the Biorobotics Laboratory, Korean University of Technology, Cheonan, Rep. of South Korea ideal scenario is then tackled in a systematic way, using the Time Domain Passivity Control Approach [4], [6], as a tool to render the communication channel passive. The methods presented can be regarded as a framework for designing teleoperation systems which allows any possible communication channel causalities and characteristics and, in general, is compatible with any control architecture, that is, of any number of channels [7], [8] and any coupling control method, e.g. [2], [4], [5], [9].

The paper generalizes previous work [6], [10], [11] conceived for specific teleoperation architectures but is aimed as a stand alone paper rather than incremental. The treatment of the communication channel has been matter of discussion in many publications. Indeed, a data channel linking two physical systems, as is the case of teleoperation, has been proved to be a source of energy and thus a cause of system instability [2]. The reasons lay into the fact that such channels are used to link physical systems but they miss physical meaning themselves. By way of illustration, a very long flexible beam connecting one mass to another could not ever become unstable (unless both masses would actively be moved in order to excite resonance frequencies). Instead, typical data channels, exchanging positions and forces for instance, implicitly reproduce the behavior of an ideal weightless infinitely rigid bar, but with delay. This is an element which cannot be physically modeled and misses therefore coherence with the rest of the system, i.e. master, slave, etc. The secular work based on the scattering parameters [2], or the wave variables formulation [3], uses a lossless transmission line model for representing the communication channel. The data channel is thus given a physical meaning, i.e. a transmission line, and benefits from its physical characteristics, as is the passive nature of such elements, even in presence of time delay.

The treatment given in this publication is based on a complete electrical representation of the system using lumped elements. Rather than considering part of the system as being composed by lumped electrical elements, as can be master, slave and controllers, and part as a transmission line, i.e. the channel with the scattering parameters formulation, the system communication channel is given the meaning of one or more electrical networks which can be directly connected to the rest of the system.

The framework involves a) the process of identification and isolation of active networks in the electrical domain and b) passivation of those networks using common Time Domain Passivity -based controllers. An example is used across the sections to facilitate the exposition of the methods and arguments presented. Section II shows how the 


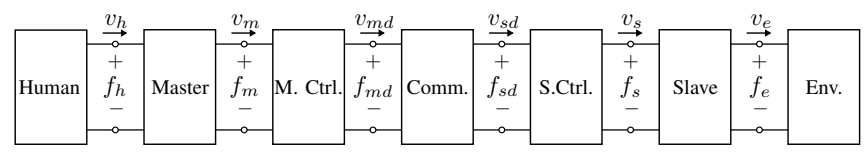

Fig. 1: Network representation of a teleoperation system.

electrical-mechanical analogy can be used to derivate the electrical and network representations. Section III addresses the ambiguity problem in the causality of some channel architectures. Section IV explores passivity of a fundamental communication element, the Time Delay Power Network (TDPN). Section $\mathrm{V}$ gives the general design guidelines. Section VI shows some experimental results for a case study.

\section{Physical Modeling: An Augmented ELECTRICAL REPRESENTATION}

Although teleoperation systems are of mechanical nature, it is often convenient to develop the system analysis in other analogous representation domains such as the electrical or the more abstract Hamiltonian port representation [12]. In general, an analogy is useful because it allows to analyze a more or less unexplored domain by means of elements and laws which belong to another, more proficient one. The conventional mechanical - electrical analogy, regarded as velocity - current analogy, maps forces into voltages and velocities into currents. The electrical representation presents benefits over the mechanical modeling in that system equations can be derived on a simple manner (e.g. by extracting Kirchoff's equations) and it enables the network representation. The network is a one step higher abstraction level which allows easy passivity analysis [2] since system passivity is guaranteed by the passivity of its subsystem connected in cascade (see Fig. 1). The passivity analysis of specific elements can be individuated and the internals of each element can be 'masked' by its energy behavior.

In order to extract such representation it is fundamental to identify the ports of each network. In turn, the ports are identified by a conjugate pair of force and velocity signals and cannot be arbitrarily chosen, but rather they must be identifiable in the electrical circuit.

The goal of this section is to find the network representation of any communication channel configuration.

\section{A. Example: The Position - Force measured Architecture}

The scheme is shown in Fig. $2^{1}$. The velocity (or position) command to the slave robot, $v_{s d}$, is the master velocity, $v_{m}$, delayed; The slave force sensor signal, $f_{e}$, is fed back to the master device.

The electrical analogue is shown in Fig. 3. In finding the network representation of the communication channel, a common mistake related to this kind of architectures is to consider the right port of the communication network by the variables $f_{e}$ and $v_{s d}$ since they are located in different parts of the scheme as can be seen in Fig. 3 but not in Fig. 2.

\footnotetext{
${ }^{1}$ For better readability, force and velocity time dependency $(t)$ is dropped in the figures.
}

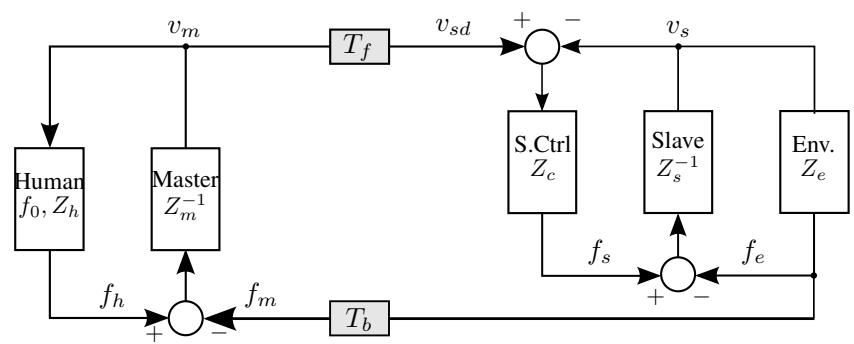

Fig. 2: Block diagram of the $\mathrm{P}$ - measured $\mathrm{F}$ teleoperation architecture.

Therefore they do not represent the communication medium. For instance, the use of that pair of signals for encoding wave variables leads to unstable systems since the modeled transmission line is carrying energy of non-power correlated signals. The electrical scheme indubitably unveils the signal pairs describing each port of the communication as:

$$
\text { Left: }\left\{\begin{array} { l } 
{ f _ { m } = f _ { e } ( t - t _ { b } ) , } \\
{ v _ { m } ( t ) . }
\end{array} \text { Right: } \left\{\begin{array}{l}
f_{s}(t), \\
v_{s d}=v_{m}\left(t-T_{f}\right) .
\end{array}\right.\right.
$$

By extracting the Kirchoff's equations of the circuit, it is possible to express $f_{e}$ as a function of $v_{m}$ and $f_{s}$, that is, the signals available at the two ports. This allows to model the communication as two-port network.

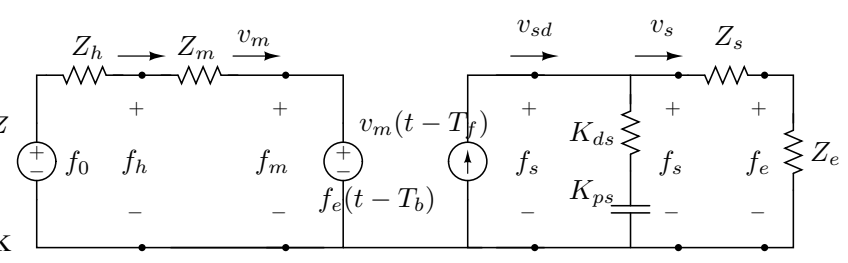

Fig. 3: Electrical scheme of a P-F measured architecture.

In the following some elements needed for the network representation are described.

\section{B. Ideal Flow and Effort Sources}

The circuit in Fig. 3 introduces ideal flow (velocity) and effort (force) dependent sources analog to current and voltage sources. In this context they represent a trajectory generator at some point of the scheme. For instance, the commanded motion by the master operator or the reflected force from the sensor.

Proposition 2.1: An ideal current source can supply and absorb unlimited power forever and so it represents an unlimited source of energy.

Proof: The current through an ideal current source is independent of the voltage across it. The internal resistance of an ideal current source is infinite ${ }^{2}$ (setting the source with zero current is identical to an ideal open circuit). The voltage at the port of an ideal current source is thus completely determined by the circuit it is connected to. When connected to an external load, for instance, the voltage across the source

\footnotetext{
${ }^{2}$ The internal resistance of a current source is modeled in parallel with the source.
} 
approaches infinity as the load resistance approaches infinity (an open circuit). The power of an ideal current source is independent from the internal resistance (due to its infinity value) and is proportional to the voltage (positive or negative) across the source.

As will be seen in upcoming sections, this is an important characteristic in the rationale behind framework here presented.

\section{Delayed Dependent Source Network Representation}

Delayed flow or effort sources are ideal sources whose value is dependent on some past signal in the circuit. Delayed sources are unsurprising in teleoperation due to the distributed master - slave nature. They are however difficult to handle because they mask the communication delay. As mentioned above, the channel is a source of energy due to the delay and therefore a cause of system instability. The following representation is proposed, which separates source and delay by shifting the source to its undelayed location and attaching it to a transport network, Time Delay Power Network (TDPN) from now on. Both representations, the delayed source and the augmented representation using a TDPN are mathematically equivalent. Fig. 4 and Fig. 5 show the models for a flow source and an effort source respectively. The equivalences for the flow source case (Fig. 4 ) are as:

$$
\begin{aligned}
v_{2}(t) & =v_{1}(t-T)=V_{o}(t-T) \\
f_{2}(t) & =f_{0}(t) \\
f_{1}(t) & =f_{2}(t-T)
\end{aligned}
$$

Similar equivalences can be extracted for the delayed effort source case. As will be seen, the benefit of this augmented

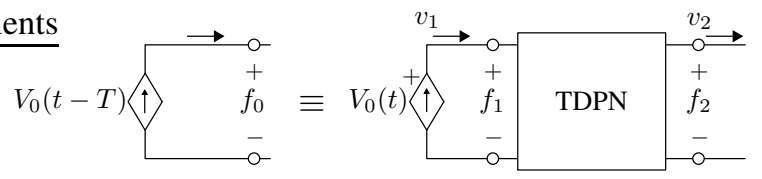

Fig. 4: Delayed current source and its extended representation.

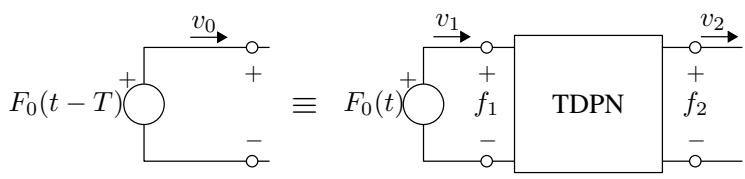

Fig. 5: Delayed effort source and its extended representation.

representation is precisely the isolation of the energy induced by the delay. An ideal source is an active element and the energy generated must be conserved (as it is responsible for the motion at some point of the system). Instead, the energy induced by the delay should not be allowable as it can violate the system passivity.

\section{Time Delay Power Networks (TDPN)}

Based on the assumption that the interaction between the communication channel and the rest of the system depends only on the terminal behavior of the channel, the Time Delay Power Network (TDPN) is defined as a communication 2port network characterized by the delay needed by the signals to travel from one terminal to the other and is completely described by a pair of power conjugated variables, force and velocity, at each port. Two causalities are possible: Velocity traveling forward - force traveling backward; and the opposite, force traveling forward - velocity backwards. Fig. 6(a) shows the block diagram representation of a communication, Fig. 6(b) the equivalent electrical representation and Fig. 6(c) the equivalent TDPN. The interest in representing a communication as a TDPN lays in that (a) it is analogous to an electrical circuit, benefiting thus from the proficiency of the electrical domain, and (b) the interconnection medium, i.e. the ports, is emphasized rather than the internals of communication, this is, the network masks a set of properties. By analyzing the energy flow displayed at the ports of the TDPN, the behavior of the communication is completely described (in what concerns passivity), irrespective of time delay, package loss, black outs and jitter, since each of these factors has an impact on the energy flow through the network.

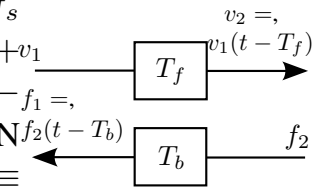

(a) Communication channel

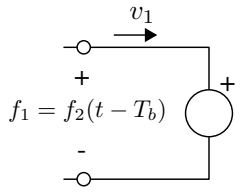

(b) Equivalent 2-port communication channel network

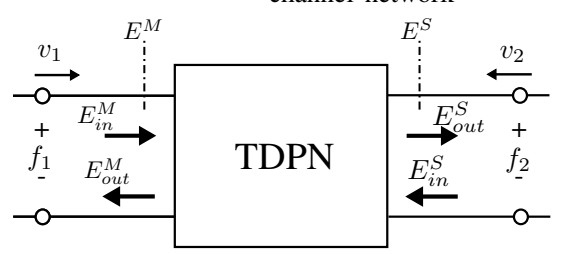

(c) Equivalent Time Delay Power Network
Fig. 6: In and out energies of the Position - Force Delay Network.

Recalling the example presented in Fig. 2 and its electrical representation in Fig. 3, the scheme with the augmented representation, that is, undelayed ideal effort and flow sources attached to TDPNs, is as shown in Fig. 7.

\section{Ambiguity of the Network CAusality}

As seen in the example in Fig. 2, the causality of the communication channel, i.e. the input/output relationships between flow and effort, is ambiguous, that is, the block diagram misleads the extraction of the network representation. This ambiguity is more obvious in other channel architectures such as a position-position or a force-force. In a position-position, for instance, the signals in the communication channel can be represented as flows (assuming position increments or velocity signals are sent instead of absolute positions). The paradox is that although energy is transmitted through the channel, the outgoing and incoming flow signals do not define the ports at each side of the communication 


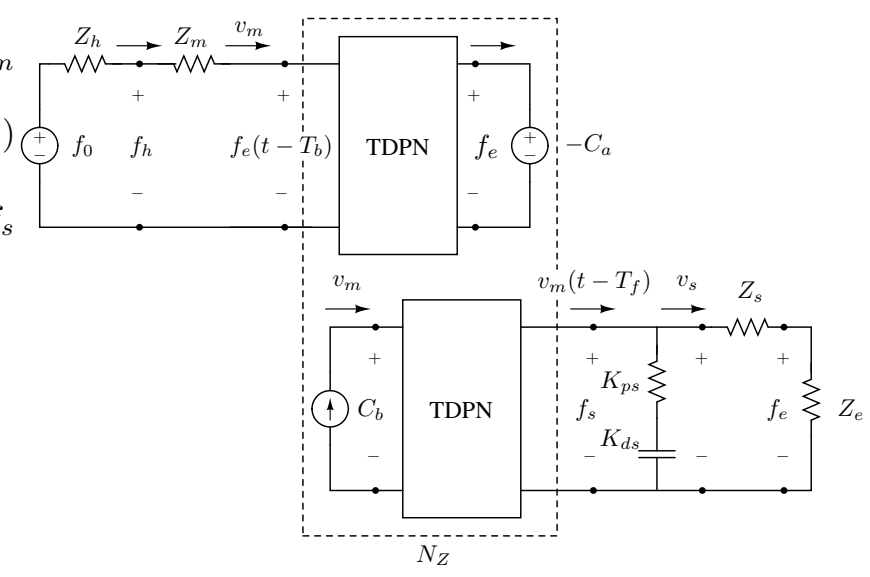

Fig. 7: Augmented electrical representation of the Position Force measured scheme.

since they are not power correlated. As such, a network representing the communication channel cannot be extracted.

A solution to solve the ambiguity in the network causalities is presented in this paper based on the un-delayed flow and effort sources attached to TDPNs networks. The employment of these elements unveils a hidden, powerport conform, a network structure, which allows the full network treatment. The scheme in Fig. 7 is an augmented, mathematically equivalent, representation of Fig. 3. By way of illustration, a diagram of a position-position channel and its augmented representation is shown in Fig. 8.

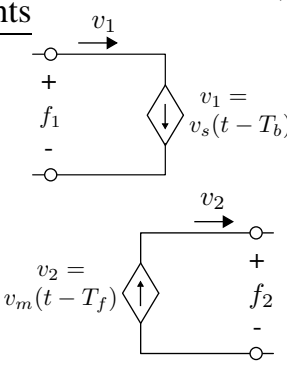

(a) Electrical scheme

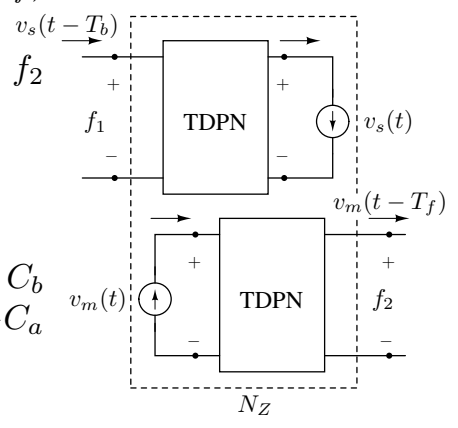

(b) Agumented network representation
Fig. 8: Position-position communcation network disambiguity.

\section{Passivity Analysis}

The tool used for stability in this framework is the Time Domain Passivity Approach (TDPA) [13]. While other stabilization methods could be here considered, the TDPA presents the advantage that besides being simple and flexible the approach emerges from an ideal case design, which is as well one of the main features of the framework in this paper. Briefly, the TDPA has two main elements: the Passivity Observer (PO), which monitors the energy flow of a network in the time domain; and the Passivity Controller (PC), which acts as a variable damper to dissipate the energy introduced by the network. In the following, the PO / PC control strategy for TDPN type of networks is reviewed.

\section{A. Passivity Observer for the TDPN}

The energy flow stored in the TDPN is given by:

$$
E^{N}(t)=E^{M}(t)+E^{S}(t), \quad \forall t \geq 0,
$$

where left and right port energy contributions, $E^{M}$ and $E^{S}$ respectively, are

$$
\begin{aligned}
E^{M}(t) & =\int_{0}^{t} P^{M}(\tau) d \tau=\int_{0}^{t} f_{1}(\tau) v_{1}(\tau) d \tau \\
E^{S}(t) & =\int_{0}^{t} P^{S}(\tau) d \tau=\int_{0}^{t}-f_{2}(t) v_{2}(t) d \tau .
\end{aligned}
$$

Being $P^{M}$ and $P^{S}$ left and right port powers. Further, an energy value exhibited at a port can be split into input energy flow entering the network, and output flow coming out from the network [10] (see Fig. 6(c)), corresponding to positive and negative contributions as:

$$
\begin{aligned}
E^{M}(t) & =E_{\text {in }}^{M}(t)+E_{\text {out }}^{M}(t), \quad \forall t \geq 0, \\
E^{S}(t) & =E_{\text {in }}^{S}(t)+E_{\text {out }}^{S}(t), \quad \forall t \geq 0 .
\end{aligned}
$$

See Appendix for derivation of (4).

Using (2), the passivity condition is given by:

$$
E^{N}(t)=E^{M}(t)+E^{S}(t) \geq 0, \quad \forall t \geq 0 .
$$

(5) can not be used straightaway in an experimental setup since both port energy values, $E^{M}$ and $E^{S}$ cannot be available at the same time due to the delay. In order to make the condition observable the following algebraic workaround ( [6], [11])is needed. Using input and output energy values as defined in (4), condition (5) can be rewritten as:

$$
\begin{aligned}
E^{N}(t) & =E_{\text {in }}^{M}(t)-E_{\text {out }}^{M}(t)+E_{\text {in }}^{S}(t)-E_{\text {out }}^{S}(t)= \\
& =E^{L 2 R}(t)+E^{R 2 L}(t) \geq 0,
\end{aligned}
$$

being

$$
\begin{aligned}
& E^{L 2 R}(t)=E_{\text {in }}^{M}(t)-E_{\text {out }}^{S}(t), \quad \forall t \geq 0, \\
& E^{R 2 L}(t)=E_{\text {in }}^{S}(t)-E_{\text {out }}^{M}(t), \quad \forall t \geq 0 .
\end{aligned}
$$

$E^{L 2 R}$ and $E^{R 2 L}$ are decoupled energy flows from left to right and from right to left respectively of the TDPN. Condition (5) holds as long both constrains, $E^{L 2 R} \geq 0$ and $E^{R 2 L} \geq 0$ are satisfied (see Appendix for proof). $E^{\bar{L} 2 R}$ and $E^{R 2 L}$ are however not yet observable due to the same reason as in (5). The passivity condition taking the time delay into account, i.e. observable is written as:

$$
\begin{aligned}
& E_{\text {obs }}^{L 2 R}(t)=E_{\text {in }}^{M}\left(t-T_{f}\right)-E_{\text {out }}^{S}(t) \geq 0, \quad \forall t \geq 0, \\
& E_{\text {obs }}^{R 2 L}(t)=E_{\text {in }}^{S}\left(t-T_{b}\right)-E_{\text {out }}^{M}(t) \geq 0, \quad \forall t \geq 0 .(8)
\end{aligned}
$$

Intuitively this means that the energy coming out from one side of the TDPN must be lower bounded by the energy flow entering the channel from the opposite side, and viceversa (see Fig. 6(c)). Proof of the passivity condition in (8) can be found in the Appendix. 
The Passivity Observer is an algorithmic extrapolation of (8) plus the correction introduced by the Passivity Controller to satisfy passivity. The two Passivity Observers are thus defined as:

$$
\begin{aligned}
W_{S}(n+1) & =E_{\text {in }}^{M}\left(n-T_{f}\right)-E_{\text {out }}^{S}(n)+E_{P C}^{S}(n), \\
W_{M}(n+1) & =E_{\text {in }}^{S}\left(n-T_{b}\right)-E_{\text {out }}^{M}(n)+E_{P C}^{M}(n),(9)
\end{aligned}
$$

where $W_{S}$ and $W_{M}$ are left and right TDPN Passivity Observes. Both $E_{P C}^{M}$ and $E_{P C}^{S}$ (later defined in (11)) are master and slave dissipated energies by both Forward Passivity Controller (FPC) and Backward Passivity Controller (BPC) respectively (see Fig. 9).

\section{B. Passivity Controller for the TDPN}

As in [13] the Passivity Controller comes in the form of a variable damping which adapts as a function of the observed energy flow, this is, if the observer reports activity, the damping coefficient, $\beta$, must be such that it dissipates that amount of active energy. In the admittance configuration (velocity is modified to produce the dissipation) the controller at the right side is described as:

$$
v_{2}(n)=\hat{v}_{2}(n)-\beta(n) f_{2}(n),
$$

where $\hat{v}_{2}(n)$ is the untouched velocity signal coming from the master. The $\beta$ coefficient can be obtained as:

$$
\beta(n)= \begin{cases}0 & \text { if } W_{S}(n)>0 \\ \frac{-W_{S}(n)}{f_{2}^{2}(n)} & \text { else, if }\left|f_{2}(n)\right|>0\end{cases}
$$

And the dissipated energy:

$$
E_{P C}^{S}(n)=\Delta T \sum_{k=1}^{n-1} f_{2}^{2}(k) \beta(n) .
$$

Applying (10) keeps $W_{S} \geq 0$, which in turn keeps $E_{\text {obs }}^{L 2 R} \geq 0$ and therefore $E^{L 2 R} \geq 0$ (6). On a similar way the PC controller for the impedance case (velocity is conserved while force is modified to produce the dissipation) can be defined. The network created by the system FPC + TDPN + BPC, as seen in Fig. 9, is thus a passive one:

$$
E_{P C}^{M}(n)+E^{N}(n)+E_{P C}^{S}(n) \geq 0, \quad \forall t \geq 0 .
$$

See [13], [14] for more detailed proof and performance analysis of the TDPA.

\section{Passivity of the delayed flow / effort source}

Recalling Fig. 4, the question arises as whether the same passivity treatment for the general TDPN can be applied to the TDPN attached to the undelayed source. Since an ideal source can absorb infinit amount of energy, as justified in Prop. 2.1, it is concluded that active energy generated at the TDPN and traveling toward the source will not affect system passivity. Thus passivity must be controlled only at the opposite port of the source (right in the case of Fig. 4). The idea is exposed in Fig. 10: the unique PC placed at the right side guarantees that the energy coming out from

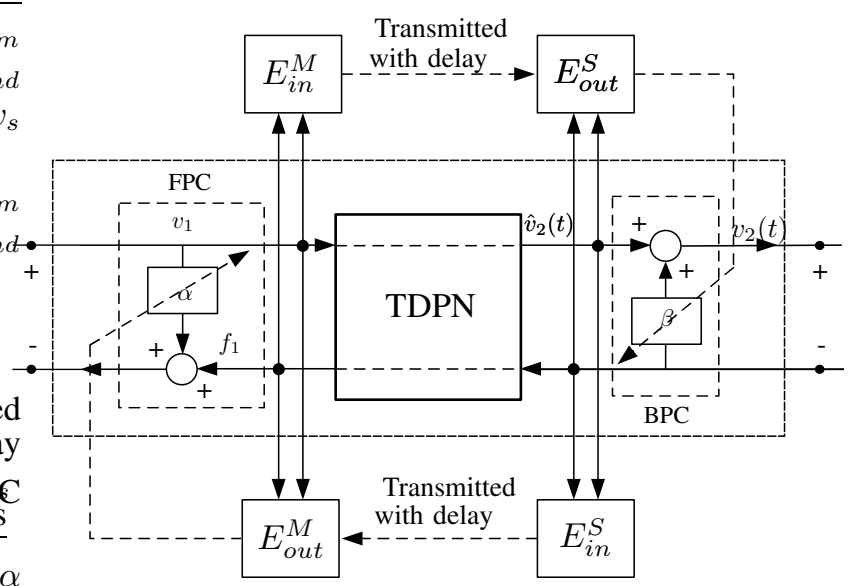

Fig. 9: Passivated TDPN using master and slave PO / PC.

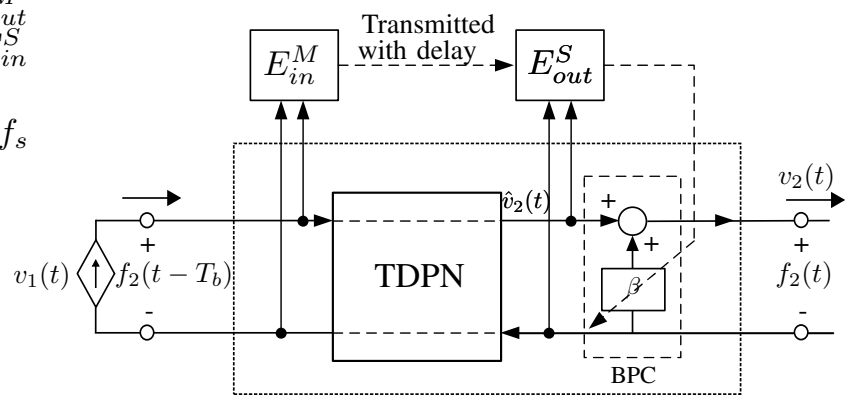

Fig. 10: The left PC is not needed due to the ideal current source.

the TDPN is lower bounded by the energy generated by the source.

Recalling the example presented in Fig. 2 and its augmented electrical representation in Fig. 7 the scheme integrating the PO/PCs is as shown in Fig. 11.

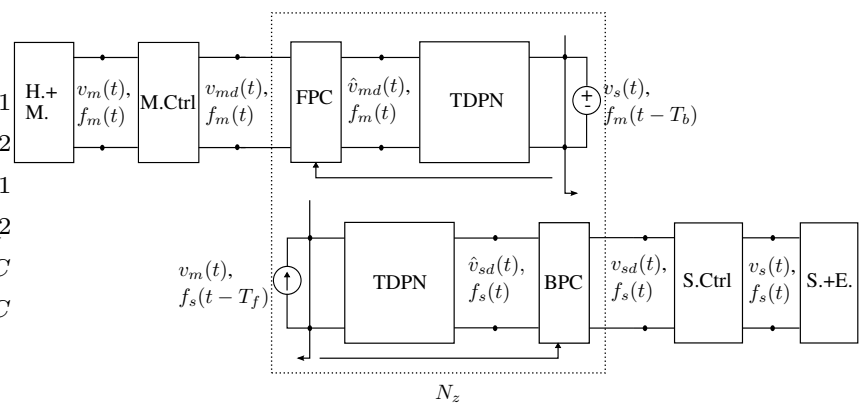

Fig. 11: The system is augmented with FPC and BPC which keep the com. channel passive.

\section{Design Guidelines}

In the previous sections, a set of tools for representing and passivating teleoperation systems have been exposed. The follwoing guidelines summarize the above and can be considered as a design methodology:

1) Design in the ideal case (no delay nor package loss) using block diagram representation. 
2) Extraction of the electrical system incorporating delay using the tools presented in Sec. II.

3) Expansion of delayed dependent sources such that they become a combination of TDPN and an undelayed ideal source as described in Sec. II-C.

4) TDPNs identification and passivity treatment by means of PO/PC blocks at the opposite side of the undelayed source as described in Sec. IV.

These guidelines will also lead to designs based on other architectures. For instance, in the Position - Position architecture the communication channel conveys position data back and forth. Although energy clearly travels from one side to the other, this type of channels do not offer intuitive network representation since only flows are being transmitted. The network description of such a communication structure will be unveiled by using the above methods. Once the network description is clear, passivity can be controlled by employing the $\mathrm{PO} / \mathrm{PC}$ rationale.

\section{EXPERIMENT}

Some experiments where performed using a pair of PHANToMs 1.5 controlled from the same computer at a sampling rate of $1 \mathrm{Khz}$. The slave Phantom was equipped with the Nano17 ATI force-torque sensor. The PD controller was parametrized for maximum performance assuming a nearly ideal case, i.e. high stiffness (P) and null damping (D). The sampling rate was set at $1000 \mathrm{~Hz}$. Overall, the bare system configuration (without any PO / PC) presented very narrow stability regions, allowing a maximum roundtrip delay of $T_{r t}=3 \mathrm{~ms}$. Fig. 12(a) shows position and force responses for a round trip delay of $100 \mathrm{~ms}, T_{f}=50 \mathrm{~ms}$ and $T_{b}=50 \mathrm{~ms}$. Fig. 12(b) shows in and out energies for each TDPN. As it can be seen, in both TDPN, the output values are higher than the input ones, showing evident active behavior. This proofs empirically the activity of the TDPN networks. Fig. 12(c) shows the energy responses after the Passivity Controllers. As can be seen the dissipative term of the PC brings the make the output values drop to the input values, keeping thus the system passive.

\section{CONCLUSION}

The time delay in a closed loop system is a complex issue which has gathered a great deal of attention. In a physical system, where the human is part of the loop, the problem becomes even more complex. In the aim of generalizing, systematizing and keeping the analysis simple, the framework here presented can be used as a tool for designing teleoperation systems with any conceivable channel causality. The framework is constructed on the basis of the TDPA, used as passivity tool. The TDPA is powerful is these contexts because it endorses the same nature as teleoperation systems, that is, time-varying and nonlinear. The Position - Force measured architecture has been used across this article as a paradigm to develop the analysis and as design example. The framework has been successfully applied in other architectures as well, such as Position - Position or the three channel, Position - Force measured - Position. Future
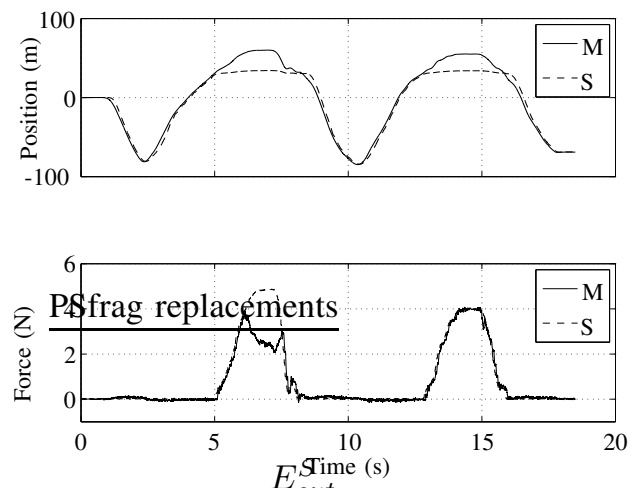

(a) Experimental data. $240 \mathrm{~ms}$ round trip delay. Contact

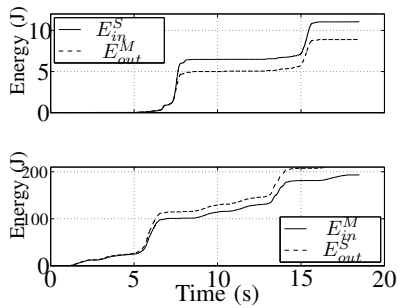

(b) Energies before PC
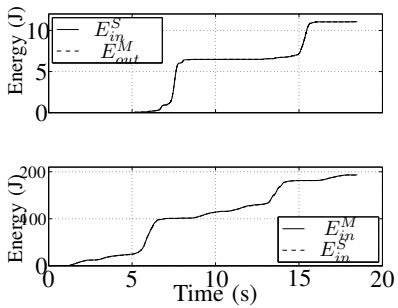

(c) Energies after PC
Fig. 12: Experimental data. $100 \mathrm{~ms}$ round trip delay. Contact

work will deal with the four channel architecture design and implementation for multi body mechanics.

\section{APPENDIX}

Proposition 1.1: The energy flow observed at a port of a network, $E$, can be split into positive and negative components, each of which indicates the direction of propagation.

Splitting positive and negative port power as:

$$
\begin{aligned}
& P_{+}(t)=P(t) \quad \forall f(t), v(t) \quad \text { s.t. } \quad f(t) v(t)>0, \\
& P_{-}(t)=-P(t) \quad \forall f(t), v(t) \quad \text { s.t. } \quad f(t) v(t)<0 .
\end{aligned}
$$

Positive and negative contributions of the energy flow are

$$
\begin{aligned}
& E_{+}(t)=\int_{0}^{t} P_{+}(t) d \tau, \quad \forall t \geq 0, \\
& E_{-}(t)=\int_{0}^{t} P_{-}(t) d \tau, \quad \forall t \geq 0,
\end{aligned}
$$

both being monotonic and positive defined.

Definition 1: Input and output components of left and right port energies are related to positive and negative power as:

$$
\begin{aligned}
& E_{\text {in }}^{M}(t)=E_{+}^{M}(t), \quad E_{\text {out }}^{M}(t)=E_{-}^{M}(t), \\
& E_{\text {in }}^{S}(t)=E_{+}^{S}(t), \quad E_{\text {out }}^{S}(t)=E_{-}^{S}(t), \quad \forall t \geq 0 .
\end{aligned}
$$

\section{A. Proof of (7).}

Theorem 1.2: If both observed energy flows are $E_{o b s}^{L 2 R}(t) \geq 0$ and $E_{o b s}^{R 2 L}(t) \geq 0$ then the system is passive. 
Proof: To check passivity means to prove (5). Since both $E_{\text {out }}^{S}$ and $E_{\text {out }}^{M}$ are monotonic the following holds:

$$
\begin{aligned}
& E_{\text {out }}^{S}\left(t-T_{b}\right) \leq E_{\text {out }}^{S}(t), \quad \forall t \geq 0, \\
& E_{\text {out }}^{M}\left(t-T_{f}\right) \leq E_{\text {out }}^{M}(t) . \quad \forall t \geq 0,
\end{aligned}
$$

Observed decoupled energy expressions, $E_{o b s}^{L 2 R}$ and $E_{o b s}^{R 2 L}$ from (8), are thus lower bounded by the decoupled real (opposed to observed) expressions, $E^{L 2 R}$ and $E^{R 2 L}$ from (7). This is:

$$
\begin{aligned}
& E_{\text {obs }}^{L 2 R}(t) \leq E^{L 2 R}(t), \quad \forall t \geq 0, \\
& E_{\text {obs }}^{R 2 L}(t) \leq E^{R 2 L}(t) . \quad \forall t \geq 0 .
\end{aligned}
$$

Therefore if constrains $E_{o b s}^{L 2 R}(t) \geq 0$ and $E_{o b s}^{R 2 L}(t) \geq 0$ are satisfied, so do (6) and thus (5).

\section{REFERENCES}

[1] M. E. V. Valkenburg, Ed., Intorduction To Modern Network Synthesis. John Wiley and Sons, Inc., 1965.

[2] R. J. Anderson and M. W. Spong, "Bilateral control of teleoperators with time delay," IEEE Transactions on Automatic Control, vol. 34, no. 5, pp. 494-501, May 1989.

[3] G. Niemeyer, "Using wave variables in time delayed force reflecting teleoperation," Ph.D. dissertation, Massachussetts Institute of Technology, Sep. 1996.

[4] J.-H. Ryu, B. Hannaford, C. Preusche, and G. Hirzinger, "Time Domain Passivity Control with Reference Energy Behavior," in Proceedings of the 2003 IEEE/RSJ Intl. Conference on Intelligent Robots and Systems, Las Vegas, Nevada, USA, October 2003.

[5] G. Raju, G. Verghese, and T. Sheridan, "Design issues in 2-port network models of bilateral remote manipulation," in IEEE International Conference on Robotics and Automation, vol. 3, no. 1316 - 1321, Scottsdale, AZ , USA, 1989.

[6] J.-H. Ryu, J. Artigas, and C. Preusche, "A passive bilateral control scheme for a teleoperator with time-varying communication delay," to appear in Elsevier Journal of Mechatronics, 2010.

[7] D. A. Lawrence, "Stability and transparency in bilateral teleoperation," IEEE Transactions on Robotics and Automation, vol. 9, no. 5, pp. 624637, 1993.

[8] K. Hashtrudi-Zaad and S. E. Salcudean, "Bilateral parallel force/position teleoperation control," Journal of Robotic Systems, 19: 155167. doi: 10.1002/rob.10030, 2002.

[9] M. Franken, B. Willaert, S. Misra, and S. Stramigioli, "Bilateral telemanipulation: Improving the complementarity of the frequency- and time-domain passivity approaches," in IEEE International Conference on Robotics and Automation, Shanghai, China, 2011.

[10] J.-H. Ryu and C. Preusche, "Stable bilateral control of teleoperators under time-varying communication delay: Time domain passivity approach," in IEEE International Conference on Robotics and Automation, Rome, Italy, April 2007.

[11] J. Artigas, J.-H. Ryu, and C. Preusche, "Time domain passivity control for position - position teleoperation architectures," to appear in Presence, Teleoopearators and Virtual Environments, 2010.

[12] C. Secchi, S. Stramigioli, and C. Fantuzzi, Control of interactive robotic interfaces A port-Hamiltonian approach, ser. Springer Tracks in advanced robotics. New York: Springer Verlag, 2007, vol. 29.

[13] B. Hannaford and J.-H. Ryu, "Time domain passivity control of haptic interfaces," in Proc. IEEE ICRA '01, 2001, pp. 1863-1869.

[14] B. Hannaford, Y. S. Kim, and J.-H. Ryu, "Sampled and continuous time passivity and stability of virtual environments," in IEEE International Conference on Robotics and Automation, vol. 1, no. 822 - 827, Taipei, Taiwan, Sep. 2003. 